Workshop on Econophysics of the

International Conference on Statistical Physics (SigmaPhi2011)

International Journal of Modern Physics: Conference Series

Vol. 16 (2012) 93-104

(C) World Scientific Publishing Company

DOI: $10.1142 /$ S2010194512007805

\title{
ESTIMATION OF FLUX BETWEEN INTERACTING NODES ON HUGE INTER-FIRM NETWORKS
}

\author{
KOUTAROU TAMURA*, WATARU MIURA ${ }^{\dagger}$ and MISAKO TAKAYASU ${ }^{\ddagger}$ \\ Department of Computational Intelligence and Systems Science \\ Interdisciplinary Graduate School of Science and Engineering, Tokyo Institute of Technology \\ 4229, Nagatsuta-cho, Midori-ku, Yokohama 226-8502, Japan \\ *tamura@smp.dis.titech.ac.jp \\ ${ }^{\dagger}$ miura@smp.dis.titech.ac.jp \\ ${ }^{\ddagger}$ takayasu@dis.titech.ac.jp \\ HIDEKI TAKAYASU \\ Sony Computer Science Laboratories \\ 3-14-13, Higashi-Gotanda, Shinagawa-ku, Tokyo,141-0022, Japan \\ takayasu@csl.sony.co.jp \\ SATOSHI KITAJIMA $\S^{\S}$ and HAYATO GOTO ${ }^{\circledR}$ \\ SPECIA Team, Industrial Survey Department, TEIKOKU DATABANK, LTD. \\ 2-5-20, Minami Aoyama, Minato-ku, Tokyo, 107-8680

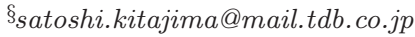 \\ 『hayato.gotou@mail.tdb.co.jp
}

\begin{abstract}
We analyze Japanese inter-firm network data showing scale-free properties as an example of a real complex network. The data contains information on money flow (annual transaction volume) between about 7000 pairs of firms. We focus on this money-flow data and investigate the correlation between various quantities such as sales or link numbers. We find that the flux from a buyer to a supplier is given by the product of the fractional powers of both sales with different exponents. This result indicates that the principle of detailed balance does not hold in the real transport of money; therefore, random walk type transport models such as PageRank are not suitable.
\end{abstract}

Keywords: Complex network; non-linear interaction; scale free.

\section{Introduction}

Studies on complex networks have ignited by the concept of preferential attachment proposed by Barabasi and $\mathrm{Albert}^{1}$, and there has been a strong trend toward researching various properties of complex networks ${ }^{2,3}$. Recently, transport models on complex networks have been attracting growing attention ${ }^{4}$. For example, random walks on complex networks are being intensively investigated following the success of PageRank, which is an index to evaluate the importance of Web pages by considering artificial random walk jumping over homepages. There are many studies 
on the transportation on complex networks for evaluating nodes and explaining the characteristic value of nodes from network topologies using mathematics and physics, both fundamentally and technically ${ }^{5-9}$; however, there have been no empirical studies on the analysis of flow among nodes in a real complex network, which is scale free.

In view of the empirical studies on complex systems and scale-free networks in the real world, business firms are attracting the attention of statistical physicists because they show various types of scale-free properties confirmed by data in the form of financial reports ${ }^{10-13}$. The cumulative distribution function of sales, employees, and degrees of trading links are known to obey power laws with power exponents of 1.0, 1.3, and 1.3-1.4, respectively. In addition, the inter-firm network has been reported ${ }^{10}$ to be a small world network with a mean pair distance of about 5 , nonlinear correlation has been confirmed between sales and link degrees ${ }^{11,13}$, and a weak negative degree-degree correlation exists as a characteristic property of the inter-firm network ${ }^{11,12}$.

In this study, we analyzed data of an inter-firm network compiled by Teikoku Databank $^{\mathrm{a}}$ (TDB) in 2011, which describes details of trading relations. This dataset contains partial information about annual transaction volumes, and we can directly observe the money flow from a buyer to a supplier of materials or services. In the next section, we explain the basic properties of the data we analyzed. In sections 3 and 4, we review the scaling properties of individual firms and network structures, respectively. In section 5 , we estimate the functional form of the interaction between a pair of firms by analyzing the money-flow data and compare the results with that of the PageRank model. Finally, we conclude with a discussion in section 6 .

\section{Dataset}

The data we analyzed were compiled by TDB in January 2011. It consists of finance time-series data (COSMOS2) and buyer-supplier relation data (CCR). There are 13 job categories in the data, and we analyzed all the data except those of the firms with jobs related to finance/insurance and governmental associations. These data were found to be unsuitable. For finance/insurance, the amount of total deposits is described in the column of sales; however, this value is quite different from the sales for ordinary firms. For governmental associations, all important values such as sales and capitals were missing.

COSMOS2 (time-series) data consist of 2,334,118 Japanese firms and their corresponding financial values (sales, capitals, profits, number of employees, job category, fiscal year, address) for 20-30 years. Some of the gross properties of this data are listed in Table 1 . We can compare these numbers with the official numbers

a TEIKOKU DATABANK, LTD. is one of the largest corporate credit research company in Japan and was established in 1900. They have built a corporate database to estimate credit risks since 1972 . 
Table 1. Summary of gross quantities in the data for most recent five years (except finance and government categories).

\begin{tabular}{c||c|c|c|c}
\hline year & total firms & $\begin{array}{c}\text { total sales } \\
\text { (trillion yen) }\end{array}$ & $\begin{array}{c}\text { total employees } \\
\text { (person) }\end{array}$ & $\begin{array}{c}\text { total capitals } \\
\text { (trillion yen) }\end{array}$ \\
\hline \hline 2005 & 1213776 & 1294 & 2478 & 124 \\
\hline 2006 & 1208060 & 1338 & 2481 & 123 \\
\hline 2007 & 1213495 & 1385 & 2508 & 127 \\
\hline 2008 & 1237443 & 1408 & 2527 & 130 \\
\hline 2009 & 1297249 & 1319 & 2563 & 135 \\
\hline
\end{tabular}

Table 2. Financial quantities for most recent five years (except finance/insurance and government categories from the Financial Statements Statistics of Corporations by Industry, Annual, Ministry of Finance, Japan).

\begin{tabular}{c||c|c}
\hline & total firms & total sales \\
\hline \hline 2005 & 2718777 & 1508 \\
\hline 2006 & 2735630 & 1566 \\
\hline 2007 & 2759279 & 1580 \\
\hline 2008 & 2774434 & 1508 \\
\hline 2009 & 2771912 & 1368 \\
\hline
\end{tabular}

announced by the Ministry of Finance, Japan in the report ${ }^{14}$ of Financial Statements Statistics of Corporations listed in Table 2. When comparing Table 2 with Table 1 , the COSMOS2 data contain about $50 \%$ of the firms and $85 \%$ of the sales of the official data each year. The firms missing in this dataset are known to be mostly very small firms with only a few workers, and we believe that the data are sufficient for investigating the statistical properties of the interaction of business firms.

The other dataset, the CCR (trade) data, consists of information on firms' trade relationships and was compiled in January 2011. The data contain 3,841,496 partnership relations among 612,133 firms and the corresponding information of each trade (e.g., name of the buyer/supplier, category of goods, etc.). The data contain 7,851 annual transaction volumes (money flow to the supplier). By removing the firms with job categories of finance/insurance and government, we eventually had $3,678,870$ business links among 604,663 firms with 7,779 links characterized quantitatively by the amount of annual money flow. More details on the CCR data are summarized in Table 3.

In the Japanese economy, firms in construction, manufacturing, and wholesale/retail trade account for $65 \%$ of all firms and 70\%-80\% trades of all observed trades. We can say that the Japanese inter-firm trades mainly consist of firms in these three categories. 
Table 3. Job categories of buyers/suppliers in CCR data.

\begin{tabular}{c||c|c|c}
\hline & total firms & $\begin{array}{c}\text { total trades } \\
\text { (as a buyer) }\end{array}$ & $\begin{array}{c}\text { total trades } \\
\text { (as a supplier) }\end{array}$ \\
\hline \hline agriculture & 2852 & 9960 & 7902 \\
\hline forestry & 299 & 642 & 832 \\
\hline fisheries & 726 & 2688 & 1609 \\
\hline mining & 1142 & 5015 & 7680 \\
\hline construction & 144463 & 733788 & 525097 \\
\hline manufacturing & 115739 & 972082 & 1050261 \\
\hline wholesale/retail trade & 192452 & 1235640 & 1573191 \\
\hline finance/insurance & 3281 & 40628 & 22857 \\
\hline real estate & 17962 & 70612 & 38233 \\
\hline transport/information/communications & 28769 & 186822 & 158633 \\
\hline electricity/gas/heat/water & 383 & 12560 & 3891 \\
\hline services & 99876 & 470438 & 448021 \\
\hline \hline government & 4189 & 100621 & 3271 \\
\hline \hline
\end{tabular}

\section{Basic Properties}

Here, we review the basic properties of individual business firms by analyzing the CCR data. As mentioned above, we analyzed all of the data except those of the finance/insurance and government categories. We have already pointed out that the distributions of sales and employees obey power laws; in particular, for sales, the exponent is close to -1 , which is called Zipf's law. We confirmed these results in the CCR data, as shown in Figs. 1 and 2. Each distribution was approximated by a power law with exponents of 1.05 and 1.3, respectively. This is consistent with the former results based on different data ${ }^{10,12,13}$. In addition, the distribution of firms' capitals is plotted in Fig. 3. This distribution has several characteristic steps and hence cannot be approximated by a power law. This deviation from a power law may have been caused by minimum capital regulation or a discrete change in tax rate as a function of the capital. The distribution of the growth rate of firms is known to obey a tent-like distribution with the peak value at unity, where the

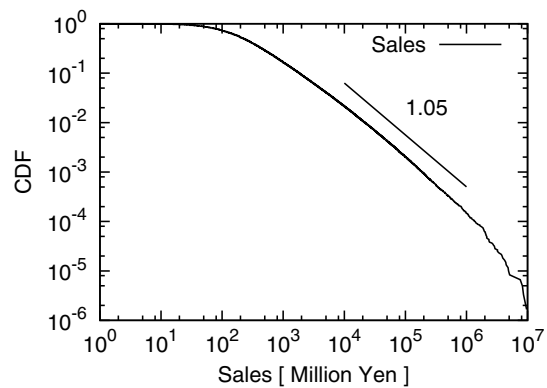

Fig. 1. Cumulative distribution of sales in $\log -\log$ scale

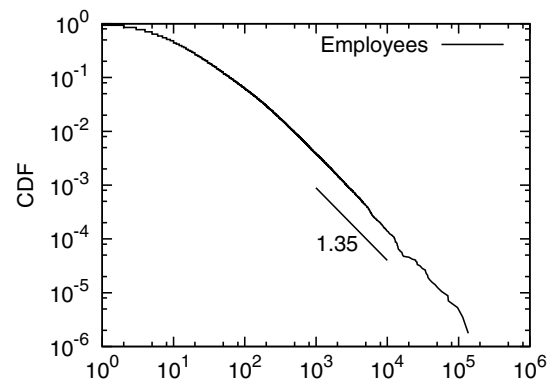

Fig. 2. Cumulative distribution of the number of employees in log-log scale 


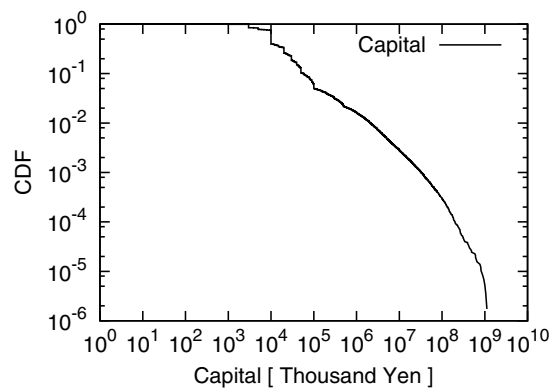

Fig. 3. Cumulative distribution of capitals in log-log scale

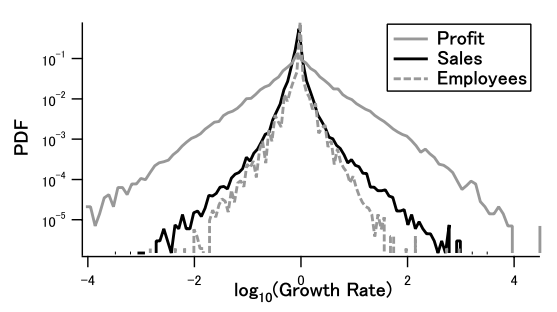

Fig. 4. Probability density of growth rate from 2009 to 2010 in $\log -\log$ scale

growth rate is defined by the value of a quantity such as sales in the specific year divided by the same quantity one year before ${ }^{13}$. The probability density of growth rates for sales, incomes, and number of employees are plotted in a semi-log plot in Fig. 4. In this plot, the Gaussian distribution is represented by a parabolic curve, and we confirmed that the growth rate probability density is higher at the center and that there are long tails in both sides for all cases, as already pointed out.

Next, we confirm the correlation between sales and the number of employees ${ }^{15,16}$. In Fig. 5, these two values are plotted in log-log scale. The points are scattered around the nonlinear relation $S \propto n^{1.3}$ for $n$ larger than $1.5 \times 10^{3}$, and the correlation coefficient is 0.50 for all the plots. The fact that the amount of sales is given by a nonlinear function of the number of employees implies that larger firms are more efficient in gathering money than smaller firms.

\section{Properties of the inter-firm network}

In general, a business transaction is made between a pair of firms, where one is a buyer and the other is a supplier, with the money flow from buyer to supplier. The CCR data contain lists of business partners for each firm with their roles, and

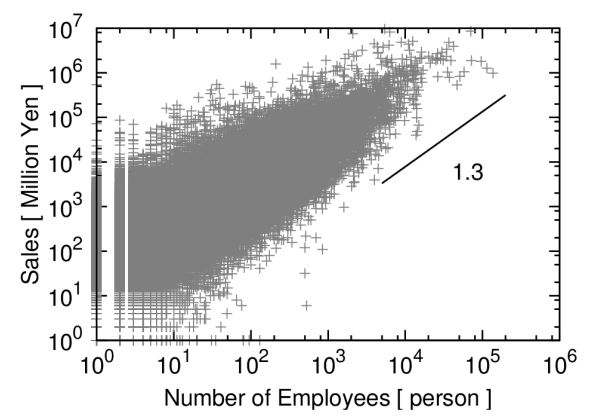

Fig. 5. Relation between sales and the number of employees 
we can draw a direct line for each pair of business partners showing the money flow, as shown in Fig. 6. The opposite direction of the link is the direction of the goods/service flow.

We can define the adjacency matrix corresponding to the money flow network as follows.

$$
A_{i j}=\left\{\begin{array}{l}
1 \text { if money flow } \mathrm{i} \rightarrow \mathrm{j} \text { exists } \\
0 \text { otherwise }
\end{array}\right.
$$

The adjacency matrix has $604,663 \times 604,663$ elements with $3,678,870$ non-zero elements, and all other matrix elements (about $3.6 \times 10^{11}$ ) are 0 ; namely, the connection is very sparse with the average number of links per node being 6.1. In Fig. 8, the cumulative distributions of link numbers for in-links and out-links, which are also called in-degrees and out-degrees, are plotted in log-log scale. Both distributions are quite similar, and they are approximated by power laws with exponents of about 1.4; this is consistent with the results already reported from the analysis of a different database in a different year ${ }^{10-13}$. As a consequence of this scale-free property, a majority of firms are transacting with only a few firms; on the other hand, there are big firms with thousands of business partners.

As a basic property of the network, we calculate the correlation coefficient defined with the following formula.

$$
r=\frac{4 M \sum_{i, j}^{N} A_{i j} k_{i} k_{j}-\left[\sum_{i, j}^{N} A_{i j}\left(k_{i}+k_{j}\right)\right]^{2}}{2 M \sum_{i, j}^{N} A_{i j}\left(k_{i}^{2}+k_{j}^{2}\right)-\left[\sum_{i, j}^{N} A_{i j}\left(k_{i}+k_{j}\right)\right]^{2}}
$$

where $M$ is the total number of links, $N$ is the total number of nodes, $k_{i}$ denotes the degree (either in-degree or out-degree) of node $i$, and $A_{i j}$ is an adjacency matrix of this network. The correlation coefficients for out-degree:out-degree, indegree:in-degree, out-degree:in-degree, in-degree:out-degree and total-degree:totaldegree (total-degree is the sum of in-degree and out-degree) were -0.047, -0.052, $-0.065,-0.034$, and -0.072 , respectively. Weak negative correlations were confirmed in this inter-firm network as reported earlier ${ }^{11,12}$.

In order to clarify this negative correlation of links, we focused on the nodes with given link numbers and observed the average link numbers of its neighbors. In Fig. 9,

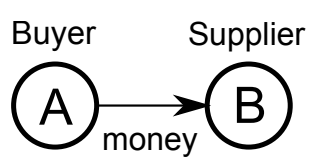

Fig. 6. Schematic view of a money flow

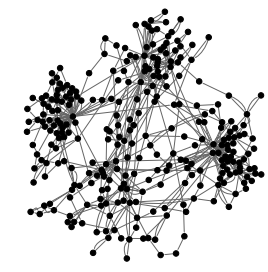

Fig. 7. Example of the inter-firm network (part of business relations in Aomori Prefecture) 


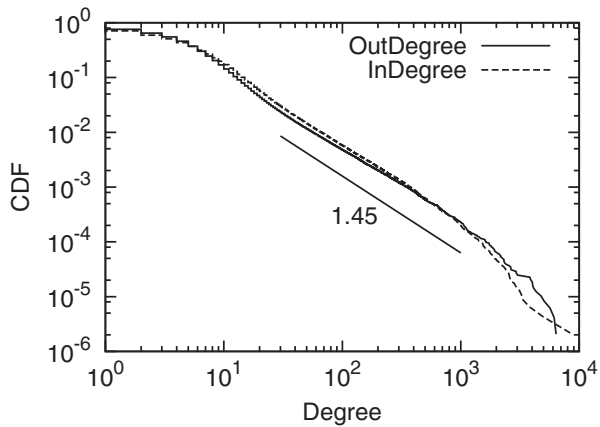

Fig. 8. Cumulative distribution of outdegree and in-degree in log-log scale

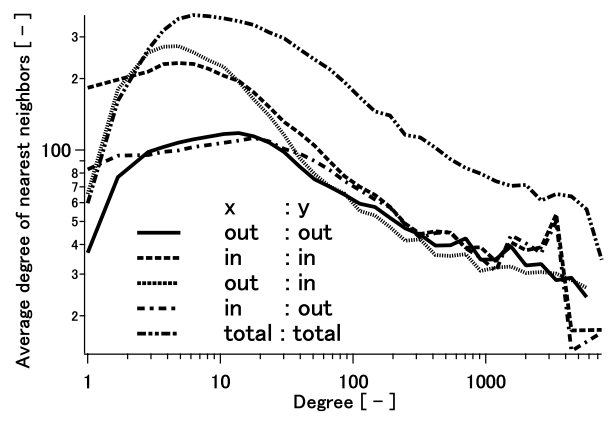

Fig. 9. Average degree of nearest neighbours vs degree

these conditional averages of the neighbors' degrees are plotted in log-log scale. As shown in this figure, larger degree nodes tend to be connected with smaller degree nodes. Link numbers are known to be strongly correlated with sales ${ }^{11}$. Figs. 10 and 11 show the scatter plots for sales and degrees in log-log scale. The correlation coefficients of sales:in-degree and sales:out-degree in log-log scale were 0.37 and 0.54 , respectively. For larger degrees, we can find a nonlinear power law relation $s_{i} \propto k_{i}^{1.3}$ like the case of employees for both in-degrees and out-degrees. Clearly, the network structure of firms plays an important role in determining the sales.

\section{Interaction between nodes}

As already mentioned, the CCR data contain detailed information on the money flow between pairs of business partners: there were data on 7,779 annual transactions involving 7,300 firms. Table 4 lists the money flow data in each job category. We analyzed this money flow data and observed the interaction among firms. As shown

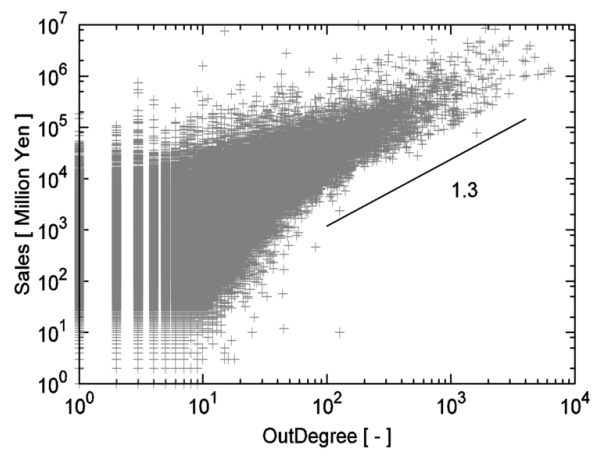

Fig. 10. Relation between sales and out-dgree

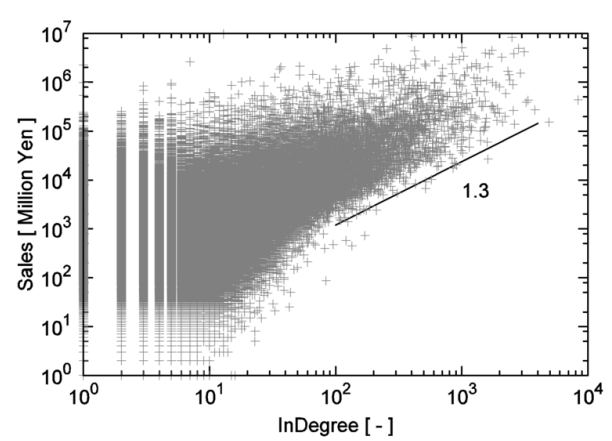

Fig. 11. Relation between sales and in-dgree 
in the table, the firms in the manufacturing and wholesale/retail categories were dominant in this data. As shown in Table 3, these job categories were also dominant in terms of overall number of firms and overall sales.

\subsection{PageRank model}

One of the most popular theoretical models of transport defined on directed complex networks is the PageRank model ${ }^{17}$. PageRank at the $i$-th node $x_{i}$ is defined by the steady solution of the following equation.

$$
x_{i}(t+1)=r \sum_{j=1}^{N} A_{j i} \frac{x_{j}}{k_{j}^{\text {out }}}+1-r
$$

where $A_{i j}$ is the adjacency matrix, $k_{i}^{\text {out }}$ is the out-degree of the $i$-th node, and $r$ is a parameter taking a value between 0 and 1 that stands for the ratio of random jump to any node. The value of PageRank in the limit of $r \rightarrow 0$ is the existence probability of a random walker on the network. PageRank was also studied analytically, and by applying the mean field approximation, it was proved to behave as

$$
x_{i} \propto k_{i}^{i n}
$$

Namely, $x_{i}$ is simply proportional to the value of in-degree, which has been confirmed numerically for the business firm network ${ }^{18}$. This result is unfavorable for PageRank to become a model of money flow on the network, because if we regard the value of PageRank to be proportional to the sales, then, the solution does not fulfill the empirical relation of Fig. 11, $S_{i} \propto\left(k_{i}^{i n}\right)^{1.3}$. It is likely that real transport between firms is not approximated by the simple random walk.

Table 4. Job categories and number of observable money flow data.

\begin{tabular}{c||c|c|c}
\hline & total firms & $\begin{array}{c}\text { total trades } \\
\text { (as a buyer) }\end{array}$ & $\begin{array}{c}\text { total trades } \\
\text { (as a supplier) }\end{array}$ \\
\hline \hline agriculture & 8 & 4 & 8 \\
\hline forestry & 0 & 0 & 0 \\
\hline fisheries & 5 & 6 & 1 \\
\hline mining & 5 & 5 & 3 \\
\hline construction & 451 & 566 & 207 \\
\hline manufacturing & 2645 & 2740 & 2665 \\
\hline wholesale/retail trade & 3136 & 3537 & 4009 \\
\hline finance/insurance & 32 & 28 & 9 \\
\hline real estate & 44 & 46 & 31 \\
\hline transport/information/communications & 380 & 353 & 483 \\
\hline electricity/gas/heat/water & 17 & 19 & 13 \\
\hline services & 549 & 517 & 417 \\
\hline government & 28 & 30 & 5 \\
\hline total & 7300 & 7851 & 7851 \\
\hline \hline
\end{tabular}




\subsection{Estimating interaction}

In order to clarify the underlying interaction among nodes, we observed the correlation between the strength of money flow for a link and characterizing quantities such as sales or link numbers at the nodes. We assumed that the money flow or flux is described by the following functional form with any function of $f$ and $g$ :

$$
f l u x(A \rightarrow B) \propto f\left(X_{A}\right) g\left(X_{B}\right)
$$

where $\mathrm{A}$ and $\mathrm{B}$ denote the buyer and supplier, respectively; the representative variable $\mathrm{X}$ actually means sales $\mathrm{S}$; capital is $\mathrm{C}$; the number of employees is $\mathrm{E}$; the in-degree is $\mathrm{I}$; and the out-degree is O. Let us estimate the functional form of $f$ and $g$ of $X_{A}$ and $X_{B}$ by observing the dependence on one variable while the other variable is fixed.

All figures listed below show the values of flux as the vertical axis and the values of a representative variable as the horizontal axis in log-log scale, while the values of fixed variables are categorized into three classes (small, medium and large). For example, in Fig. [12], the values of flux are plotted as a function of buyer's sales, while the supplier's sales are categorized. Here, the symbol $S_{B} 10^{4}$ [MYen] represents the selected data points where the supplier's sales $S_{B}$ are approximately $10^{4}$ million yen. The dotted straight lines in these figures show theoretical guide lines representing power law functions that fit well with the plots.

In the case of sales, we can find rather clear power law relations in the smallerscale range of sales for both buyers and suppliers, as seen in Figs. 11 and [12]. As for the number of employees, we can also find rough power law relations, as seen in Figs. 13 and 14, although these relations look less clear for categories with small numbers of employees. For the capital shown in Figs. 15 and [16], no remarkable relation can be observed. From Figs. 17 to 20, we can find power law relations for categories with large degrees; however, the relations are not clear for categories with small degrees. Summarizing these results, we propose that the best combination of

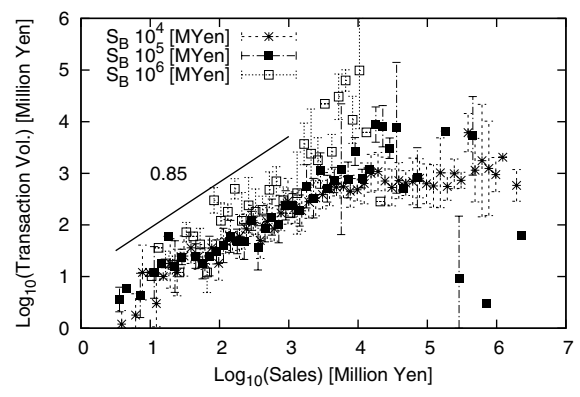

Fig. 12. Relation between money flow and buyer's sales in log-log scale with fixed supplier's sales

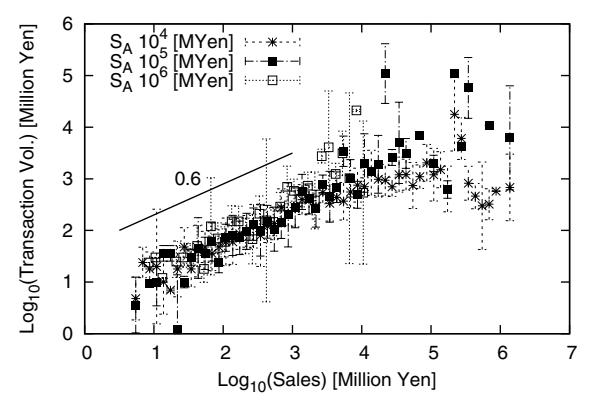

Fig. 13. Relation between money flow and supplier's sales in log-log scale with fixed buyer's sales 


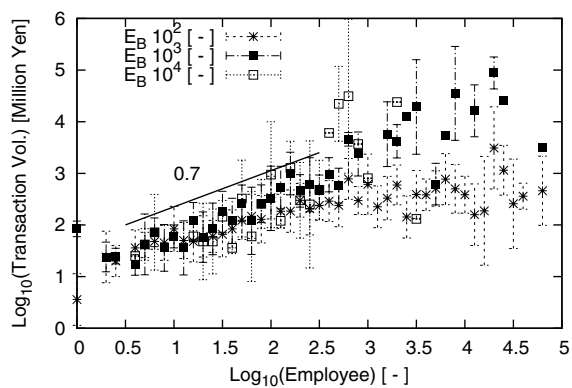

Fig. 14. Relation between money flow and buyer's employees in log-log scale with fixed supplier's employees

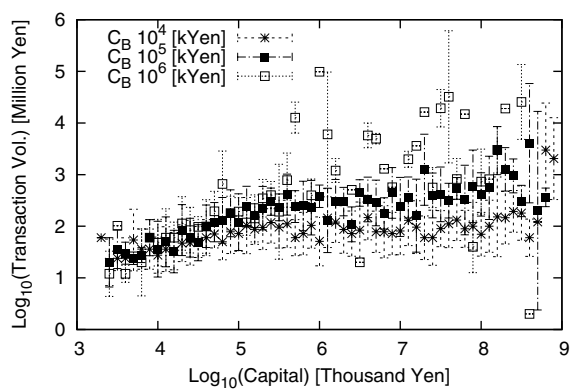

Fig. 16. Relation between money flow and buyer's capitals in log-log scale with fixed supplier's capitals

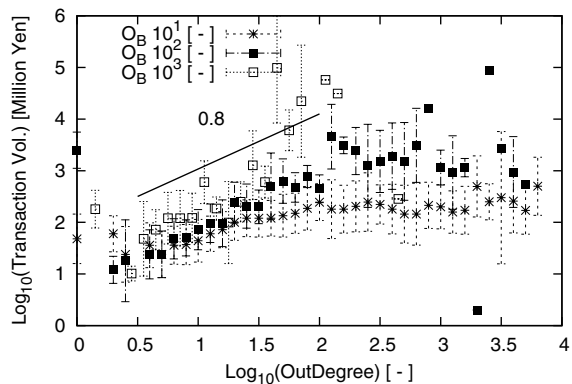

Fig. 18. Relation between money flow and buyer's out-degree in log-log scale with fixed supplier's out-degree

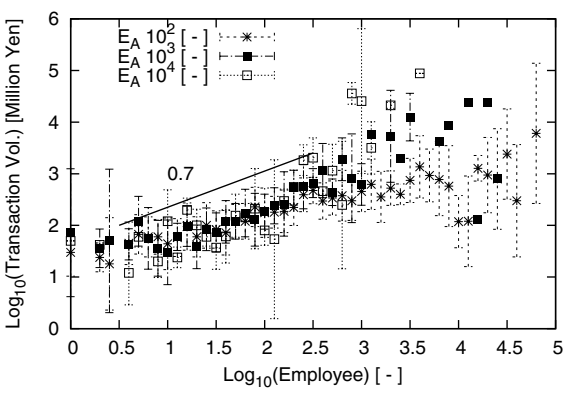

Fig. 15. Relation between money flow and supplier's employees in log-log scale with fixed buyer's employees

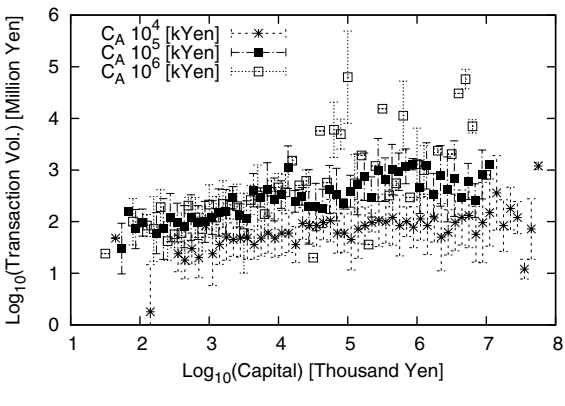

Fig. 17. Relation between money flow and supplier's capitals in log-log scale with fixed buyer's capitals

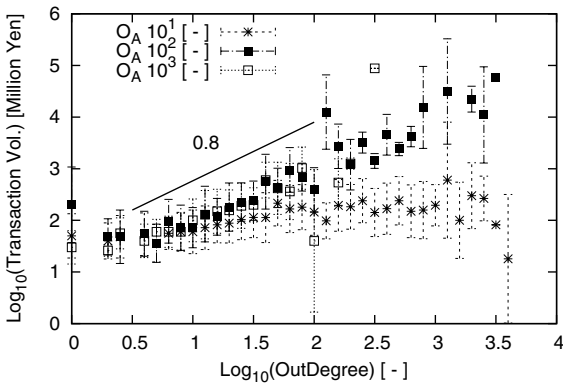

Fig. 19. Relation between money flow and supplier's out-degree in log-log scale with fixed buyer's out-degree 


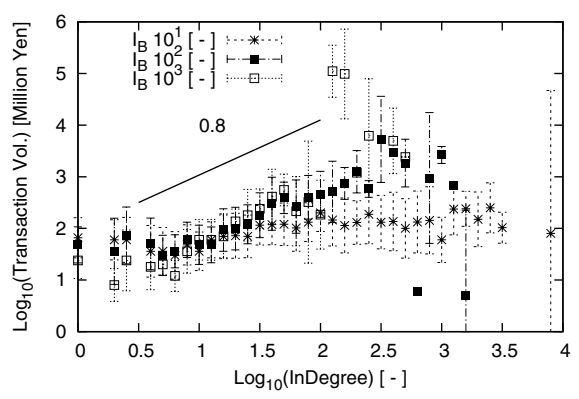

Fig. 20. Relation between money flow and buyer's in-degree in log-log scale with fixed supplier's in-degree

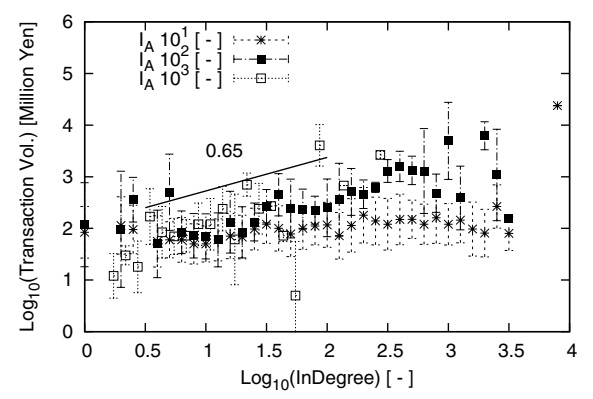

Fig. 21. Relation between money flow and supplier's in-degree in log-log scale with fixed buyer's in-degree

representative variables is sales at the nodes, and the flux of money from a buyer of sales $S_{A}$ to a seller of sales $S_{B}$ is given as

$$
f l u x(A \rightarrow B) \propto S_{A}^{\alpha} S_{B}^{\beta}
$$

where the values of exponents are $\alpha=0.85$ and $\beta=0.65$, as estimated by fitting.

The functional form of flux given in the form of Eq.(6) is familiar in the fields of international economy and population sociology by the name of generalized gravity model; it is named so because it is analogousness with Newton's law of gravity 8,9 . In these models, the amount of trade between two countries of GDPs $S_{A}$ and $S_{B}$ or traffic between two cities of population $S_{A}$ and $S_{B}$ is assumed to follow Eq.(6). Therefore, our results are not remarkable in terms of economy and sociology. Also, it is easy to show that in the special case of $\alpha \rightarrow 1, \beta \rightarrow 0$, the flux is equivalent to the case of a random walk on the network, namely, the case of PageRank. In this case, the amount of flow from a buyer node is proportional to the sales $S_{A}$, and all flows from this node are of the same amount, which is realized in the case of pure diffusion.

\section{Conclusion}

We analyzed the strength of interaction among nodes in the inter-firm network by observing large amounts of data of business firms. The relation we found is described by Eq.(6), which shows that the flux or annual money flow from a buyer firm to a supplier firm is proportional to the products of fractional powers of both sales with different exponents. The general form of flux given by Eq.(6) includes PageRank with the special case of $\alpha \rightarrow 1, \beta \rightarrow 0$. The values of the exponents that we obtained from the data $(\alpha=0.85, \beta=0.65)$ are far from these values, indicating that the transport of money in the business firm network is far from the thermal diffusive transport in equilibrium. 
In the near future, our task is to clarify the properties of network systems in which transport is governed by a form of Eq.(6). As this system is not in thermal equilibrium, we may expect various types of new behaviors.

\section{Acknowledgments}

This work is partly supported by Grant-in-Aid for challenging Exploratory Research 22656025, Japan Society for the Promotion of Science(JSPS)

\section{References}

1. A.-L. Barabasi and R. Albert, Science 286,509-512, (1999).

2. N. Masuda and H. Kori, Virtual Journal of Biological Physics Research 20, 10, (2010).

3. T. Hasegawa and K. Nemoto, Phys. Rev. E 81, 051105, (2010).

4. A. Baronchelli and R. Pastor-Satorras, PhysRevE 82, 011111, (2010).

5. S. Brin and L. Page, Computer Networks and ISDN Systems 30, 107-17, (1998).

6. J. D. Noh and H. Rieger, PhysRevLett 92,118701, (2004).

7. B. Tadić, G. J. Rodgers and S. Thurner, International Journal of Bifurcation and Chaos, Vol.17, No.7, 2363-2385, (2007).

8. R. Feenstra, Princeton Univ. Press Chapter.5, 137-173, (2004).

9. R. Piermartini and L. Rousova, WTO Staff Working Paper, ERSD-2008-06, (2008).

10. M. Takayasu, S. Sameshima, T. Ohnishi, Y. Ikeda, H. Takayasu, K. Watanabe, Annual Report of the Earth Simulator Center April 2007 - March 2008, 263 (2008).

11. Y. Fujiwara and H. Aoyama, Eur. Phys. J. B, 77, 565-580, (2010).

12. H. Watanabe, H. Takayasu and M. Takayasu, arXiv:1111.4852v1 [q-fin.GN].

13. T. Ohnishi, H. Takayasu and M. Takayasu, Progress of Theoretical Physics Supplement, No.179, 157, (2009).

14. Ministry of Finance Japan, Financial Statements Statistics of Corporations by Industry, Annual, : http://www.mof.go.jp/english/pri/reference/ssc/index.htm

15. K. Okuyama, M. Takayasu and H. Takayasu, Physica A 269, 125-131, (1999)

16. H. Watanabe, H. Takayasu and M. Takayasu, Statistical Studies on Interrelationships of Financial Indicators of Japanese Firms, The Physical Society of Japan 2009 Autumn Meeting, in Japanese.

17. T. Ohnishi, H. Takayasu and M. Takayasu, IPSJ SIG Technical Reports 2010-MPS81, 1-3 (2010)

18. K. Tamura et al., Growth-rate distribution by Gravity Interaction Model and time evolution of the Interfirm network, The Physical Society of Japan 2011 Autumn Meeting, in Japanese. 